\title{
Teleconsulta: nova fronteira da interação entre médicos e pacientes
}

\author{
Teleconsultation: new frontier of the interaction between doctors and patients \\ Teleconsulta: nueva frontera de la interacción entre médicos y pacientes \\ Carlos André Aita Schmitza , Marcelo Rodrigues Gonçalvesa, Roberto Nunes Umpierrea, Ana Célia da Silva Siqueirab, \\ Otávio Pereira D’Ávila ${ }^{b}$, Cynthia Goulart Molina Bastos ${ }^{b}$, Rafael Gustavo Dal Morob, Natan Katz ${ }^{\mathrm{b}}$, Erno Harzheimª
}

\section{Resumo}

A teleconsulta, uma ação de telessaúde, apesar de ser regulamentada em vários países, ainda não é permitida no Brasil. O presente texto explora a situação da teleconsulta na América do Norte, na Europa e em outros países, fazendo um paralelo com a situação nacional dentro da medicina e de outras profissões da saúde. Ao final, por meio do referencial utilizado, é construída uma argumentação de forma a assumir um posicionamento favorável à regulamentação da teleconsulta no país.

\section{Abstract}

Teleconsultation, a telehealth action, although regulated in several countries, is still not allowed in Brazil. This text explores the topic of teleconsultation in North America, Europe and other countries, parallel to the national reality in medicine and other health professions. Finally, from the theoretical framework analyzed, an argument is built to assume a favorable position to the regulation of teleconsultation in the country.

\section{Resumen}

La teleconsulta, una acción de telesalud, a pesar de ser regulada en varios países, todavía no es permitida en Brasil. El presente texto explora el tema de la teleconsulta en América del Norte, en Europa y en otros países, haciendo un paralelo con la realidad nacional en la medicina y otras profesiones de la salud. Finalmente, a partir del marco teórico analizado, se construye una argumentación para asumir un posicionamiento favorable a la regulación de la teleconsulta en el país.
Palavras-chave:

Telemedicina

Ética Clínica

Consulta Remota

Brasil

Keywords:

Telemedicine

Ethics, Clinical

Remote Consultation

Brazil

Palabras clave:

Telemedicina

Ética Clínica

Consulta Remota

Brasil
Como citar: Schmitz CAA, Gonçalves MR, Umpierre RN, Siqueira ACS, D'Ávila OP, Bastos CGM, et al. Teleconsulta: nova fronteira da interação entre médicos e pacientes. Rev Bras Med Fam Comunidade. 2017;12(39):1-7. http://dx.doi.org/10.5712/rbmfc12(39)1540
Fonte de financiamento: declaram não haver. Parecer CEP: não se aplica. Conflito de interesses: declaram não haver. Procedência e revisão por pares: revisado por pares. Recebido em: 30/05/2017.

Aprovado em: 17/11/2017. 


\section{Introdução}

As ações de telessaúde podem ser entendidas como uma interação a distância mediada por Tecnologias da Informação e Comunicação (TICs), entre pessoas e/ou equipamentos, de forma síncrona ou assíncrona e com finalidade assistencial ou educacional, conforme ilustrado no Quadro 1.1-3

Quadro 1. Lista de ações em telessaúde, por tipo de interação, modalidade e sincronicidade. TelessaúdeRS/UFRGS, Porto Alegre, 2015.

\begin{tabular}{|lllll|}
\hline Ação de telessaúde & \multicolumn{1}{l}{ Interação a distância mediada por TIC entre } & Modalidade & Sincronidade \\
\hline Teleconsulta & profissional de saúde & paciente & assistencial & síncrona/assíncrona \\
Teleconsultoria & profissional de saúde & profissional de saúde & assistencial & síncrona/assíncrona \\
Telediagnóstico & equipamento de coleta & profissional de saúde & assistencial & assíncrona/síncrona \\
Telecirurgia & profissional de saúde & equipamento robótico de cirurgia & assistencial \\
Telemonitoramento & sensor de coleta & dispositivo de monitoramento e & assistencial & síncrona/assíncrona \\
& & armazenamento & educacional síncrona/assíncrona \\
Teleducação & profissional(is) de saúde & profissional(is) de saúde & educacional & assíncrona \\
Teleducação & profissional(is) de saúde & Objeto de Aprendizagem - AO & educacional & assíncrona \\
Teleducação & profissional de saúde & Segunda Opinião Formativa - SOF & &
\end{tabular}

A telessaúde insere-se na prática diária, ao menos, de três maneiras: a) como uma nova forma de entregar práticas tradicionais, como no caso da teleconsultoria e da teleducação; b) com caráter substitutivo e aditivo (principalmente em locais antes sem acesso), como no caso do telediagnóstico e da teleconsulta; e c) como um metasserviço que permeia e apoia tanto as ações de saúde como os fluxos de pessoas e informações dentro de um sistema de saúde, por meio do uso combinado das várias ferramentas de telessaúde (por exemplo, no apoio ao complexo regulador). ${ }^{4-7}$

No que diz respeito à teleconsulta, ela pode ocorrer por serviços seguros de telefonia, videoconferência, chat, e-mail, mensagens instantâneas e aplicativos para dispositivos móveis. Dados da atenção primária indicam que $66 \%$ dos pacientes telefonam para seus médicos para tranquilização, aconselhamento ou esclarecimento sobre sintomas preocupantes..$^{5,8}$

Nesse sentido, em 2014 foi realizado um ensaio clínico randomizado por clusters e com avaliação econômica de triagem telefônica na atenção primária (o maior até a data da sua publicação), com 21.000 pacientes de 42 clínicas inglesas alocados em três grupos: cuidado usual, triagem telefônica por médico e triagem telefônica por enfermeiro auxiliado por sistema de apoio à decisão. Os resultados mostraram incrementos, no número de atendimentos, de $33 \%$ e $48 \%$ nos grupos triados por médicos e enfermeiros, respectivamente, mas com custos equivalentes ao grupo de cuidado usual. O aumento do acesso foi atingido sem prejuízo da segurança e da satisfação dos pacientes. ${ }^{9}$

Apesar de sua ubiquidade, a teleconsulta ainda não é permitida no Brasil. O presente texto explora esse fato, fazendo um paralelo com a situação nacional, tanto para a medicina quanto para outras profissões da saúde. Ao final, por meio do referencial utilizado, é construída uma argumentação de forma a assumir um posicionamento favorável à regulamentação da teleconsulta no país. 


\section{Teleconsulta na América do Norte}

Os EUA estão bastante avançados na discussão da teleconsulta e da telemedicina. Pela legislação aprovada até dezembro de 2015, a questão maior não é sobre permitir a teleconsulta, mas se um médico de um estado pode atuar no mercado de um médico de outro estado: ${ }^{10}$

- A maioria dos 50 estados exige registro profissional no estado de exercício a distância e não apenas no estado de origem;

- Apenas três estados adotam regras de reciprocidade de registro e outros nove permitem registro estendido ou condicional;

- Como no Brasil, também ocorre a consulta entre pares (teleconsultoria).

O segundo ponto é a regulamentação da consulta virtual (teleconsulta):

- Dezenove estados a permitem sem restrições;

- Dos demais 31, apenas 3 exigem uma consulta presencial inicial, um exige paridade 1:1 e os outros ao menos um atendimento presencial em algum momento;

- Somente três estados exigem a presença de outro profissional de saúde (médico ou não) junto ao paciente no momento da interação virtual.

Como terceiro ponto, a prescrição por internet geralmente é permitida e regrada em todos os estados, com algumas exceções para medicações de indução ao aborto. ${ }^{10}$

O Canadá utiliza a teleconsulta por meio de telefonia ou internet da mesma forma para áreas remotas e rurais como para urbanas. ${ }^{11}$

\section{Teleconsulta na Europa}

Na Europa, 24 dos 28 países membros possuem legislação sobre teleconsulta: ${ }^{7}$

- Dezessete países permitem a teleconsulta, dos quais 3 com restrições (emergências, áreas com carência de médicos, necessidade de primeira consulta presencial), 11 possuem legislação específica e 3 (Finlândia - desde 1997, Noruega e Espanha) consideram as ações de saúde e de telessaúde legalmente equivalentes;

- Três países não permitem a teleconsulta (Alemanha, Eslováquia e Itália);

- Quatro estão em fase de estudo (Áustria, Escócia, Grécia e Polônia).

\section{Teleconsulta em outros países}

O México foi o primeiro país da América Latina com investimento em telemedicina e permite a teleconsulta para pacientes que vivem em comunidades rurais a partir de 2001. ${ }^{12} \mathrm{Na}$ Austrália a teleconsulta é permitidade desde 1994 e no Japão foi liberada em 1997.13,14 


\section{Teleconsulta no Brasil}

A lei 12.551, de 15 de Dezembro de 2011, determina que o trabalho presencial é equivalente ao trabalho a distância e como tal deve ser remunerado. Um dos primeiros posicionamentos do Conselho Federal de Medicina (CFM), a Resolução 1.643/2002, baseou-se na Declaração de Tel Aviv/1999 e considerava a autonomia do médico na decisão de utilizar telemedicina e a possibilidade de assistência sem contato direto com o paciente.

Restrições foram impostas pela primeira vez no Decreto Lei 4.113 de 1942, depois na quarta versão (1965) do Código de Ética Médica (artigo 5, alínea g) e reiteradas pela Resolução CFM 1.931/2009 (Código de Ética Médica, sexta versão), que em seu artigo 37 veda ao médico a prescrição de tratamento ou outros procedimentos sem exame direto do paciente, salvo casos de urgência/emergência, mas com atendimento presencial subsequente obrigatório.

Isso é reforçado pela Resolução CFM 1.974/2011, que veda ao médico a consultoria a pacientes e familiares em substituição à consulta presencial, estendendo a proibição de consulta, diagnóstico e prescrição às redes sociais ou qualquer meio de comunicação em massa ou a distância.

Essa Resolução, no entanto, em seu texto explicativo refere que: “(...) O médico pode, porém, orientar por telefone pacientes que já conheça, aos quais já prestou atendimento presencial, para esclarecer dúvidas em relação a um medicamento prescrito, por exemplo".

Outro atenuante é dado em relação ao diagnóstico, pois a Resolução CFM 2.107/2014 regulamenta e permite a emissão de laudo radiológico a partir de exame coletado a distância, desde que o paciente autorize a transmissão de suas imagens e dados clínicos. Essa resolução serve de paradigma para as demais atividades de telediagnóstico.

Mas o maior avanço ocorre em 2017, com a publicação do parecer CFM 14/2017, que permite o uso do aplicativo WhatsApp ${ }^{\odot}$ e plataformas similares (protegidas por criptografia de ponta a ponta, com chaves de 256 bits não quebráveis por métodos atuais) para comunicação entre médicos e seus pacientes, bem como entre médicos, para enviar dados e tirar dúvidas, desde que mantido o caráter confidencial. Contudo, permanece vedada a substituição de consultas presenciais pelas virtuais.

\section{Outras profissões}

- A Enfermagem permite a execução de prescrição médica a distância somente em casos de urgência ou emergência (Resolução COFEN № 0487/2015);

- A Fonoaudiologia permite apoio por avaliação a distância, mas com profissional fonoaudiólogo presente junto ao paciente (Resolução CFFa no 366/2009);

- APsicologia apresenta a legislação mais avançada, pois em 2012 regulamentou e detalhou várias modalidades de serviços psicológicos a distância, tanto em caráter clínico quanto de pesquisa (Resolução CFP № 011/2012). 


\section{Posicionamento}

Em 2016, o Conselho Federal de Medicina convidou a sociedade civil organizada para contribuir na reforma do Código de Ética Médica, declarando que os avanços e inovações científicas e tecnológicas convocam para uma reformulação orgânica do referido código. Embora o parecer CFM 14/2017 seja explícito na manutenção da proibição da teleconsulta, há uma abertura para discussão e espera-se que resoluções editadas a partir desse parecer evoluam no assunto.

O TelessaúdeRS-UFRGS, sendo um dos centros de excelência brasileiros em telessaúde, com mais de 100.000 pessoas removidas das filas de espera do SUS pela discussão de casos por telefonia (teleconsultoria), dentre vários resultados da mesma monta em outras ações de telessaúde,$^{15}$ posiciona-se favorável ao debate e regulamentação da teleconsulta no país.

Assim como EUA e Canadá, o Brasil é um país de proporções continentais, com grandes heterogeneidades e lacunas no que diz respeito à concentração de serviços de saúde e de profissionais médicos. Um dos grandes trunfos da telessaúde é a ampliação e facilitação do acesso a serviços de saúde, que, por sua vez, é um atributo essencial para o bom funcionamento de um sistema de saúde. Trabalhos de ecologia do cuidado médico mostram que cerca de $20 \%$ da população demanda mensalmente por serviços de saúde próximos à sua residência e que menos de $1 \%$ necessita de atendimento hospitalar terciário. ${ }^{16}$ Além disso, entre $80 \%$ e $90 \%$ da demanda ambulatorial são passíveis de remissão espontânea, o que permite a aplicação de condutas conservadoras e expectantes (conceito de demora permitida). ${ }^{17}$

A falta de acesso e a heterogeneidade da qualidade do primeiro nível de atenção (Atenção Primária à Saúde -APS) leva à sobrecarga dos níveis secundário (assistência especializada) e terciário (assistência hospitalar). No Brasil, as internações por causas sensíveis à APS são responsáveis por um terço das internações públicas e representaram $17,4 \%$ do custo anual com internações hospitalares no Sistema Único de Saúde em 2013 (1,97 bilhão de reais)..$^{18}$ Portanto, barreiras de acesso têm altos custos financeiros para os sistemas de saúde e grande impacto na morbimortalidade e na qualidade de vida da população. Da mesma forma, o acesso aos serviços de telessaúde impacta na qualidade dos serviços prestados, visto que as orientações fornecidas são baseadas nas melhores evidências científicas e adaptadas à realidade do SUS. Levando em conta a saúde suplementar, o acesso a serviços de saúde em tempo hábil é mandatório para minimizar a sinistralidade.

Quanto às limitações impostas pela avaliação não presencial, a clínica sempre será soberana. Médicos devem estar habilitados para prover cuidado com todos os recursos disponíveis no momento em que uma pessoa demanda por atendimento. Seja em um hospital terciário com alta densidade tecnológica, seja num posto de saúde com poucos recursos, no domicílio do paciente ou em qualquer via pública, seja a distância e mediado por tecnologia.

Os recursos disponíveis ao profissional determinam o leque de possibilidades diagnósticas e terapêuticas a ser utilizado e oferecido pelo mesmo. No que concerne à teleconsulta, médicos são treinados e possuem experiência para tomar essa decisão e assumir a responsabilidade pelo atendimento prestado. Isso é bastante claro em países como a Finlândia, onde a legislação que rege a telemedicina é a mesma que rege a medicina e onde é gerado responsabilização pelo não atendimento em tempo hábil, seja ele presencial ou a distância. 
Dentre as vantagens da telessaúde está a segurança para o paciente. Dados eletrônicos, armazenados e distribuídos dentro das normas de segurança e interoperabilidade já definidas para o país possuem um grau de privacidade muito maior do que prontuários físicos, ao que se somam as largas possibilidades de auditoria em relação aos acessos. ${ }^{19,20}$ Além disso, estudos nacionais mostram cerca de $90 \%$ de consentimento dos usuários, após esclarecimento, para o compartilhamento de seus dados entre os profissionais que os atendem. ${ }^{21}$

A regulamentação da teleconsulta no Brasil também é uma necessidade para os médicos. Tirar esse procedimento da informalidade permitirá a justa valorização financeira pelo tempo dispendido aos pacientes fora da consulta presencial e possibilitará vários arranjos de otimização de tempo e de custos, com vantagens para médicos e pacientes, como hoje já é possível para psicólogos e seus pacientes.

É fundamental que a sociedade e a categoria médica entendam que a teleconsulta é só mais um formato de prestação de serviços, e provavelmente, para algumas das especialidades médicas, a principal forma de trabalho em um futuro próximo (como já acontece com a radiologia).

Por fim, com o aumento do acesso, ampliam-se as possibilidades de seleção e de direito de escolha do paciente por profissionais médicos habilitados, ao mesmo tempo em que é gerado ganho de qualidade e redução de custos pelo aumento de escala.

\section{Referências}

1. Bashshur RL, Shannon GW, Smith BR, Alverson DC, Antoniotti N, Barsan WG, et al. The empirical foundations of telemedicine interventions for chronic disease management. Telemed J E Health. 2014;20(9):769-800. DOI: http://dx.doi.org/10.1089/tmj.2014.9981

2. World Health Organization - WHO. Telemedicine: opportunities and developments in member states. Geneva: WHO; 2010.

3. Sood S, Mbarika V, Jugoo S, Dookhy R, Doarn CR, Prakash N, et al. What is telemedicine? A collection of 104 peer-reviewed perspectives and theoretical underpinnings. Telemed J E Health. 2007;13(5):573-90. DOI: http://dx.doi.org/10.1089/tmj.2006.0073

4. Brasil. Ministério da Saúde. Manual de Telessaúde para Atenção Básica/Atenção Primária à Saúde. Brasília: Ministério da Saúde; 2012.

5. Montgomery A, Hunter D, Blair E, Hendricksen M. Telemedicine Today: The State of Affairs. 2015. [acesso 2017 Nov 30]. Disponível em: https://altarum.org/sites/default/files/uploaded-publication-files/TELEMEDICINE-State\%20of\%20Affairs_030615.pdf DOI: http://dx.doi. org/10.1089/tmj.2015.9997

6. H.R. 3750 (113th): Telehealth Modernization Act of 2013. To promote the provision of telehealth by establishing a Federal standard for telehealth, and for other purposes. [acesso 2016 Dez 6]. Disponível em: https://www.govtrack.us/congress/bills/113/hr3750

7. European Commission. Competitive and Innovation Programme. UNIversal Solutions in Telemedicine Deployment for European HEALTH care (Grant Agreement No 325215). [S.I.]: European Commission; 2013.

8. Darkins AW, Carey MA. Telemedicine and telehealth: Principles, policies, performance and pitfalls. New York: Springer; 2000.

9. Campbell JL, Fletcher E, Britten N, Green C, Holt T, Lattimer V, et al. The clinical effectiveness and cost-effectiveness of telephone triage for managing same-day consultation requests in general practice: a cluster randomised controlled trial comparing general practitionerled and nurse-led management systems with usual care (the ESTEEM trial). Health Technol Assess. 2015;19(13). [acesso 2016 Dez 6]. Disponível em: https://www.journalslibrary.nihr.ac.uk/hta/hta19130\#/abstract

10. Latoya T, Capistrant G. State Telemedicine Gaps Analysis: Physician Practice Standards \& Licensure. Washington: American Telemedicine Association; 2014.

11. Care Committee. Vancouver Division of Family Practice. Telemedicine White Paper. Vancouver: Vancouver Division of Family Practice; 2014.

12. International Telecommunication Union. Mejores prácticas de liderazgo, innovación y gestión pública en e-salud: Los casos de Brasil, México y Perú. Geneva: ITU; 2014. [acesso 2017 Nov 30]. Disponível em: https://www.itu.int/en/ITU-D/Regional-Presence/Americas/ Documents/PBLCTNS/20140331-ehealth-SP.pdf 
13. Mitchell JG. The uneven diffusion of telemedicine services in Australia. J Telemed Telecare. 1999;5 Suppl 1:S45-7. DOI: http://dx.doi. org/10.1258/1357633991932504

14. Hasegawa T, Murase S. Distribution of telemedicine in Japan. Telemed J E Health. 2007;13(6):695-702. DOI: http://dx.doi.org/10.1089/ tmj.2007.0013

15. TelessaúdeRS-UFRGS. Núcleo de Telessaúde da Universidade Federal do Rio Grande do Sul. Porto Alegre: TelessaúdeRS-UFRGS. Telessaúde Transparência; 2017. [acesso 2017 Nov 30]. Disponível em: https://www.ufrgs.br/telessauders/transparencia

16. Green LA, Fryer GE Jr, Yawn BP, Lanier D, Dovey SM. The ecology of medical care revisited. N Engl J Med. 2001;344(26):2021-5. DOI: http://dx.doi.org/10.1056/NEJM200106283442611

17. Kloetzel K. O Diagnóstico Clínico: Estratégia e Táticas. In: Duncan BB, Schmidt MI, Gugliani ERJ, Duncan MS, Giugliani C. Medicina ambulatorial: condutas de atenção primária baseadas em evidências. 4ª ed. Porto Alegre: Artmed; 2013.

18. Souza DK, Peixoto SV. Estudo descritivo da evolução dos gastos com internações hospitalares por condições sensíveis à atenção primária no Brasil, 2000-2013. Epidemiol Serv Saúde. 2017;26(2):285-94.

19. Brasil. Ministério da Saúde. Portaria № 2.073, de 31 de agosto de 2011. Regulamenta o uso de padrões de interoperabilidade e informação em saúde para sistemas de informação em saúde no âmbito do Sistema Único de Saúde, nos níveis Municipal, Distrital, Estadual e Federal, e para os sistemas privados e do setor de saúde suplementar. Brasília: Diário Oficial da União; 2011.

20. Brasil. Conselho Federal de Medicina. Sociedade Brasileira de Informática em Saúde. Manual de Certificação para Sistemas de Registro Eletrônico em Saúde (S-RES). Certificação 2013. Brasília: Conselho Federal de Medicina; 2013.

21. Becker Sander G, Medeiros Borges M, do Prado Fay JH, Costa D, Cesar Gadelha Vieira A, Bucciolli Guernelli M, et al. Health Interoperability into Practice: Results of the Development of a Consent Form in a Pilot Project in a Health District in São Paulo, Brazil. Stud Health Technol Inform. 2015;216:1007.

a Universidade Federal do Rio Grande do Sul (UFRGS). Porto Alegre, RS, Brasil. ycaaos@yahoo.com.br (Autor correspondente); marcelo.goncalves@telessauders.ufrgs.br; rnumpierre@gmail.com; ernoharz@terra.com.br

b TelessaúdeRS-UFRGS. Porto Alegre, RS, Brasil. anacelia.siqueira@telessauders. ufrgs.br; otavio.davila@telessauders.ufrgs.br; cynthia.bastos@telessauders.ufrgs.br; tsrs-rafael.moro@ufrgs.br; natan.katz@telessauders.ufrgs.br 